\title{
INVENTÁRIO DA ARBORIZAÇÃO URBANA DO MUNICÍPIO DE SOCORRO - SP E PROPOSTA DE UM ÍNDICE DE DANOS À INFRA-INFRAESTRUTURA DAS CIDADES
}

\author{
Richieri Antonio Sartori ${ }^{1}$; Ana Paula Balderi ${ }^{2}$ \\ (recebido em 21.10.2010 e aceito para publicação em 15.12.2011)
}

\section{RESUMO}

As cidades concentram $80 \%$ da população brasileira, muitas vezes de forma inadequada causando grandes problemas. A arborização urbana, quando feito de forma correta atenua alguns efeitos negativos como, poluição sonora, visual, atmosférica e hídrica. Os objetivos deste trabalho foram quantificar e qualificar a vegetação urbana do município de Socorro SP, sugerindo formas mais adequadas e ecológicas para a arborização urbana e propor uma forma de quantificar as espécies que causam maiores danos ao calçamento e fiação. Foram percorridas todas as ruas e praças do limite urbano do município e registrados todos os indivíduos arbóreos que possuíam no mínimo $50 \mathrm{~cm}$ de altura. Foi criado um índice de danos causado por cada espécie na arborização e proposta uma lista de espécies que podem ser utilizadas na arborização para ruas e praças. Foram encontradas ao todo 6829 árvores, pertencente a 48 famílias, 118 gêneros e 148 espécies. As famílias com maior quantidade de espécies foram Fabaceae e Myrtaceae. As espécies mais freqüentes foram Largestroemia indica L.e Murraya panículata (L.) Jack, 36 espécies causaram algum tipo de dano, sendo Hovenia dulcis Thumb que teve mais alto índice de danos. A conclusão foi que o município de Socorro precisa de uma grande reestruturação em sua arborização.

Palavras-chave: Vias públicas; Fiação; Calçamento; Índice de danos.

\footnotetext{
${ }^{1}$ Doutorando em Botânica, Escola Nacional de Botânica Tropical, Jardim Botânico do Rio de Janeiro, CEP 22460-030, Rio de Janeiro, RJ, Brasil. chesesartori@yahoo.com.br

${ }^{2}$ Bióloga, Associação Ambientalista Copaíba, CEP 13960-000, Socorro, SP, Brasil
}

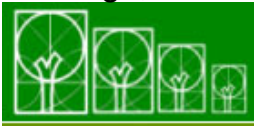




\title{
URBAN FOREST INVENTORY OF SOCORRO - SP AND PROPOSAL OF AN INDEX OF DAMAGE TO THE INFRASTRUCTURE OF CITIES
}

\begin{abstract}
Cities concentrate $81 \%$ of the Brazilian population, often in inappropriate way, causing major problems. Urban forestry, when properly done, mitigates some negative effects such as noise, visual, atmospheric and water pollution. The aim of this study was to quantify and qualify the tree vegetation in Socorro - SP, suggesting more proper and ecological ways for urban forestry and propose a way to quantify the species that cause the major damages to the pavement and electrical wiring. All the streets and squares of the urban limit were traveled, registering all trees with at least $50 \mathrm{~cm}$ tall. A damage index caused by each species was created and a list of species that can be used for afforestation in the streets and squares was proposed. In total, 6829 trees were found belonging to 48 families, 118 genera and 148 species. Families with the greatest number of species were Myrtaceae and Fabaceae. The most frequent species were Largestroemia indica $L$ and Murraya paniculata (L) Jack, 36 species have caused some damage, and Hovenia dulcis Thumb had the highest damage rate. The conclusion was that Socorro municipality needs a major restructuring in its forest.
\end{abstract}

Keywords: Public ways; Pavement; Wiring; Damage index.

\section{INTRODUÇÃO}

Nos últimos anos, as cidades passaram a concentrar grande parte da população, obedecendo a uma tendência que atualmente é de mais de $81 \%$ da população brasileira habitando nas cidades (IBGE, 2010). Esse aumento populacional provocou um crescimento da estrutura urbana, na maioria das vezes de forma inadequada, sem planejamento, o que acarretou algumas modificações no sistema natural, como a impermeabilização do solo por pavimentação e construções, provocando intenso aumento da poluição atmosférica, hídrica, visual e sonora. A arborização urbana, a implantação de florestas urbanas, áreas verdes e, 
ou, jardins são medidas que atenuam os efeitos citados anteriormente, uma vez que proporcionam uma série de vantagens, como a melhoria na qualidade do ar, absorvendo o gás carbônico e liberando oxigênio; o efeito quebra-vento; a melhoria do aspecto estético e visual; estabilidade microclimática e, por conseguinte, conforto térmico; sombreamento; a absorção de ruídos, o que reduz a poluição sonora, abrigo e alimento para pássaros (REZENDE, 1997; MELLO FILHO, 1985).

Uma árvore isolada pode transpirar, em média, 400 litros de água por dia, produzindo um efeito refrescante equivalente a cinco condicionadores de ar com capacidade de 2.500 kcal cada, funcionando 20 horas por dia. Este vapor se mistura com as partículas de poluição do ar, e quando se acumulam em nuvens, caem em forma de chuva. Além do mais, este vapor ajuda a equilibrar o clima da região (GUZZO, 1993).

Mesmo condicionando tantos benefícios, de acordo com Lima (1993), as árvores de ruas e avenidas, no geral, continuam sendo danificadas, mutiladas ou mesmo eliminadas. Nos municípios brasileiros, de modo geral, a arborização não tem um planejamento prévio, o que decorre sérios problemas de manejo. Arborizar é uma atividade onerosa e requer um planejamento adequado, para evitar correções futuras, em razão disso, o plantio de árvores no espaço urbano, segundo Gonçalves (1999), já não pode ser realizado de forma amadorística. Em calçamentos, a arborização é bastante complexa, pois as condições urbanas interferem muito no desenvolvimento das espécies (SILVA et al., 2008). Segundo Fostad e Pedersen (1997) em uma pesquisa de árvores em parques e nos centros urbanos em Oslo, Noruega, as árvores de rua possuíam saúde inferior às dos parques.

Além destes problemas, existe a crescente substituição da flora nativa por plantas exóticas, alterando o ambiente natural que resta nos centros urbanos (GOYA, 1994), e constitui uma ameaça a vegetação natural do local, devido à introdução de espécies exóticas e competidoras (CEMIG, 1997). O uso de espécies nativas poderia auxiliar para que a vegetação urbana funcionasse como um corredor ecológico, ligando fragmentos naturais e diminuindo o impacto das cidades no ecossistema como um todo (DOUROJEANNI, 1997). As árvores exóticas, muitas vezes, não possuem predadores naturais, o que pode multiplicar sem controle, tornando-se uma praga, como é o caso do pinus. Além do mais na Mata Atlântica existem espécies das mais variadas formas e flores não sendo preciso o uso de espécies exóticas (SMMARP, 1996).

O propósito deste presente trabalho foi quantificar e qualificar a vegetação urbana do município de Socorro - SP, sugerindo forma mais adequada e ecológica para a arborização urbana, bem como a prevenção e agressões e a estrutura física.

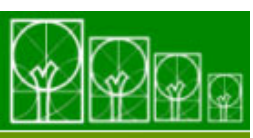

$\mathbf{S} \cdot \mathbf{B} \cdot \mathbf{A} \cdot \mathbf{U}$ Soc. Bras. de Arborização Urbana 


\section{MATERIAL E MÉTODOS}

Local de estudo - O presente trabalho foi realizado no município de Socorro, Nordeste do Estado de SP, divisa com o sul de Minas Gerais, coordenadas 22 32 ' 41"S e 46 $34^{\prime}$ '10" W e altitude média de 789 metros (Figura 1). A vegetação da região é classificada como floresta estacional semidecidual montana (VELOSO et al., 1991). O clima da região é do tipo temperado úmido, Cfb, segundo a classificação de Köppen, com verão ameno e úmido e inverno seco. A temperatura média anual é de $18{ }^{\circ} \mathrm{C}$ e precipitação média anual de $1400 \mathrm{~mm}$, com máxima de $288,7 \mathrm{~mm}$ em janeiro e fevereiro e mínima de $25.06 \mathrm{~mm}$ de março a setembro (SOCORRO, 2010). Atualmente, o município tem como principais fontes econômicas a indústria têxtil e o turismo, então o bom planejamento e adequação da estrutura urbana, assim como a arborização são de grande importância para o município (SOCORRO, 2010).

Figura 1. Localização do município de Socorro ao norte do estado de São Paulo Figure 1. Location of Socorro city, northern São Paulo State

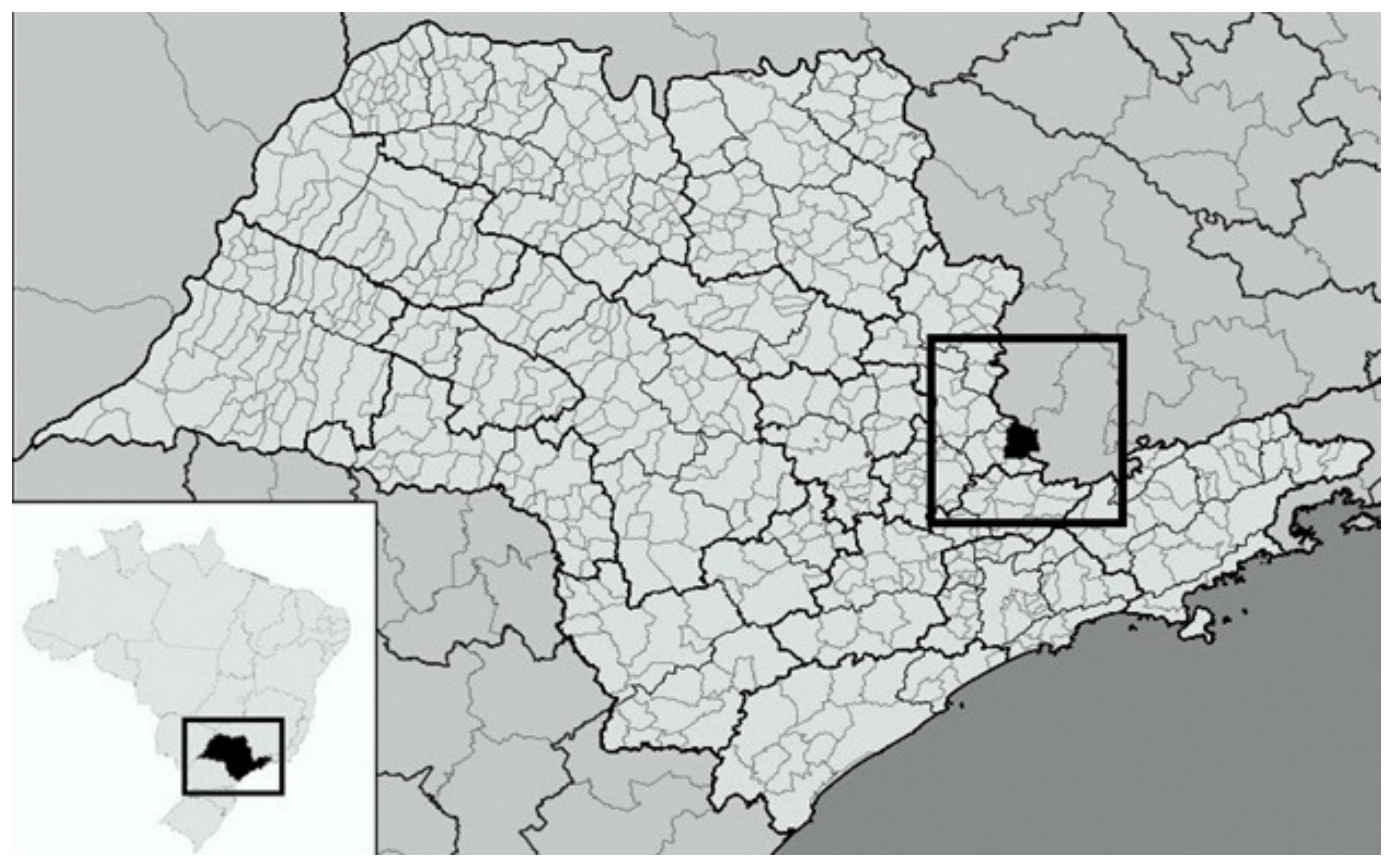

Levantamento da vegetação - Foi realizado em 14 dias, tendo início e fim no mês de fevereiro de 2009. Neste período foram percorridas todas as 259 ruas e 16 praças do limite urbano do município. Foram registrados todos os indivíduos arbóreos que possuíam no 
mínimo $50 \mathrm{~cm}$ de altura, não foram registrados aqueles que constavam em área verde, parques, estradas, matas ciliares, reservas ou jardins particulares.

Das plantas encontradas, foram registradas as espécies, local e agressões à fiação e ao calçamento. A classificação quanto às agressões foi realizada de forma visual, com nota um, sem nenhuma agressão até a nota máxima cinco, que indicava extrema agressão, destruindo calçamentos, casas ou/e a fiação prejudicada. Neste último caso foi indicado que o indivíduo fosse retirado do local e trocado por uma espécie que causasse menos danos. No caso do indivíduo estar estável, ou seja, não esteja crescendo mais como antes, o melhor a ser feito é adaptar as calçadas e a fiação.

As notas intermediárias indicavam alguma agressão, assim como dois, pequena agressão ao calçamento ou encostar-se à fiação; três, agressão evidente, porém que não causa danos, somente pequenos levantamentos de calçamentos ou encostar na fiação de forma pouco agressiva e quatro, levantamentos de calçamentos, rachaduras em muros e danificações em fiações em locais que não causem ameaças, a ponto de ser necessário o corte das mesmas ou sendo possível o manejo das mesmas.

As identificações foram realizadas através de comparação com exsicatas já identificadas existentes no Herbário da Escola Superior de Agricultura "Luiz de Queiroz" (ESALQ) e/ou através de consultas a monografias taxonômicas, especialistas e obras clássicas. Foram eliminadas as espécies que não possuem hábito arbóreo ou arbustivo e observadas às sinonímias taxonômicas. Indivíduos mortos foram contabilizados. Para classificação dos táxons foi utilizado o sistema de APG III (2009). As informações das espécies foram retiradas da lista de espécies da flora brasileira (FORZZA et al., 2010), do catálogo treeatlan (OLIVEIRA-FILHO, 2010) e da literatura de LORENZI (2000, 2002). Para confirmação da grafia das espécies e nome dos respectivos autores foi também usado o site do Missouri Botanical Garden (www.mobot.org) e Brummitt e Powell (1992).

Fator de danos - Foi criado um índice que mede o fator de destruição causado por cada espécie na arborização urbana. Este índice está baseado na soma da quantidade relativa dos indivíduos de cada espécie que causa dano ao calçamento e fiação, multiplicado pelo determinado nível de agressão, tanto para fiação como para calçamento, utilizando as fórmulas abaixo: 
Tabela 1. Descrição das fórmulas utilizadas para cálculo de danos das espécies existentes arborização urbana no município de Socorro - SP

Table 1. Description of the formula used to calculate damage caused by species used in Socorro - SP urban forest

\begin{tabular}{|l|l|}
\hline Fórmulas: \\
\hline Densidade espécie por nível de dano & $\mathrm{Da}=\sum \mathrm{i}$ \\
\hline Densidade relativa por espécie & $\mathrm{Dr}=\mathrm{Da} / \mathrm{Ti}{ }^{\star} \mathrm{Nd}$ \\
\hline Valor absoluto de calçamento & $\mathrm{Ca}=\sum \mathrm{Dr}\left(\mathrm{c} 2^{\star} 2+\mathrm{c}^{\star} 3+\mathrm{c} 4^{\star} 4+\mathrm{c} 5^{\star} 5\right)$ \\
\hline Valor relativo de calçamento & $\mathrm{Cr}=\mathrm{Ca} / \sum \mathrm{ca}^{*} 100$ \\
\hline Valor absoluto de fiação & $\mathrm{Fa}=\sum \mathrm{Dr}\left(\mathrm{f} 2^{\star} 2+\mathrm{f3}^{\star} 3+\mathrm{f} 4^{\star} 4+\mathrm{f} 5^{\star} 5\right)$ \\
\hline Valor relativo de fiação & $\mathrm{Fr}=\mathrm{Fa} / \sum \mathrm{fa}^{*} 100$ \\
\hline Índice de danos & $\mathrm{ID}=(\mathrm{Cr}+\mathrm{Fr}) / 2$ \\
\hline
\end{tabular}

Onde: Da - Quantidade de indivíduos de cada espécie i em um determinado nível; Dr Quantidade relativa de indivíduos que causam dano multiplicado pelo determinado nível; Ti - Total de indivíduos da espécie i; Nd - Nível de agressão, variando de 2 a 5, nível um, não representa agressão por isso é tido como neutro; Ca - Soma de todos os Dr da espécie i, nos quatro níveis de danos para calçamento; Fa - Soma de todos os $\mathrm{Dr}$ da espécie i, nos quatro níveis de danos para fiação; $\mathrm{Cr}$ - Valor relativo de danos da espécie i para o calçamento; Fr - Valor relativo de danos da espécie i para fiação; ID - Índice de danos.

A utilização deste índice deve ser feita somente com as espécies que possuem mais de cinco indivíduos, para que haja um número suficiente de indivíduos para a caracterização, como sendo agressiva ou não.

Sugestão de espécies - Ao final deste trabalho foi proposta uma lista de espécies que poderão ser utilizada na arborização urbana, para ruas e nas praças. As espécies foram sugeridas de acordo com sua origem, sendo prioritariamente nativas, para que a arborização urbana possa integrar-se de forma menos agressiva aos fragmentos naturais vizinhos e evitar que, de alguma forma haja um desequilíbrio causado por espécies invasoras. Também serão analisadas espécies já propostas por outros autores e observadas em outros trabalhos como adequadas para estes fins. 


\section{RESULTADOS E DISCUSSÕES}

No município de Socorro, segundo dados da Organização SOS Mata Atlântica (2009), resta apenas cerca de $3 \%$ da vegetação natural de Mata Atlântica, sendo que o histórico do município foi de grande devastação pelo plantio de café e batata. Com isso, a vegetação urbana poderia ocorrer de forma que o transtorno da devastação e desta transição entre zona urbana e rural não fosse tão drástica, podendo ser uma reserva de vegetação, onde alguns animais, principalmente pássaros e insetos, poderiam sobreviver. Os corredores ecológicos poderiam ser possíveis, principalmente em cidades de pequeno porte, onde existem fragmentos próximos aos centros urbanos, porém a vegetação deveria ser mais semelhante às naturais da área. No entanto, esta realidade está longe de ser concreta no município, pois somente $30 \%$ das espécies são nativas.

Foram registrados todos os indivíduos arbóreos presentes nos calçamentos das 259 ruas e 16 praças do município, sendo encontradas 6829 árvores, pertencente a 48 famílias, 118 gêneros e 148 espécies (Tabela 2). As sete famílias com maior quantidade de espécies representaram 52\% do total, que foram Fabaceae (32), seguida de Myrtaceae (12), Arecaceae (10), Bignoniaceae (8), Euphorbiaceae, Moraceae e Sapindaceae (5). Os gêneros que tiveram maior número de espécies foram Handroanthus com quatro espécies, seguidas de Psidium, Ficus, Euphorbia e Cassia com três. Esses cinco gêneros representaram $14 \%$ das espécies e $10,4 \%$ dos indivíduos.

As dez espécies mais encontradas representaram $67,8 \%$ do total de indivíduos, sendo estas Largestroemia indica com $25 \%$ dos indivíduos, seguidos de Murraya paniculata (20\%), Handroanthus chrysotrichus (4,5\%), Poincianella pluviosa (3,5\%), Largestroemia speciosa (3,4\%), Hibiscus rosa-sinensis (2,6\%), Callistemon imperiallis (2,5\%), Tibouchina granulosa (2,45\%), Eugenia uniflora (2,27\%) e Bauhinia divaricata (1,65\%). De todas as espécies e indivíduos encontrados, 68,2\% e 83,5\%, respectivamente, são exóticos.

Foram sugeridas para corte de 55 indivíduos, destes 36 estão mortos e 19 são indivíduos, que estão causando de alguma forma danos graves a fiação ou/e ao calçamento. Das espécies, 69,46\% do total, possuíam no máximo dez indivíduos e 17 destas, 11,5\% do total possuem apenas um indivíduo. Poucas espécies que podem ser utilizadas para a alimentação humana foram encontradas, somente 22, no total de 603 indivíduos, 8,9\% do total, com grande destaque para as mirtáceas, como pitangueira, uvaieira, goiabeira, jambo, jambolão, jabuticabeira e araçazeiro.

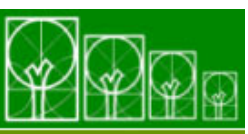

S $\cdot \mathbf{B} \cdot \mathbf{A} \cdot \mathbf{U}$ Soc. Bras. de Arborização Urbana 
Tabela 2. Espécies registradas em levantamento de espécies utilizadas na arborização urbana do município de Socorro - SP. As espécies estão organizadas em nome popular, Ori - Origem, podendo ser e - exótica ou $\mathrm{n}$ - nativa; $\mathrm{N}$, que é a quantidade absoluta; e $\mathrm{P}$, que é quantidade relativa de indivíduos encontrados

Table 2. Survey of species used in the urban forest of Socorro - SP. Species are sorted by popular name, ori - Original with e - exotic and $\mathrm{n}$ - native, $\mathrm{N}$ is the absolute quantity, and $P$, the relative amount of individuals found

\begin{tabular}{|c|c|c|c|c|}
\hline Espécie & Nome popular & Ori & $\mathbf{N}$ & $\mathbf{P}$ \\
\hline \multicolumn{5}{|l|}{ Agavacee } \\
\hline $\begin{array}{l}\text { Yucca elephantoides Regel } \\
\text { Anacardiaceae }\end{array}$ & Pata-de-elefante & e & 4 & 0,06 \\
\hline Mangifera indica $\mathrm{L}$. & Manga & e & 44 & 0,61 \\
\hline Schinus molle L. & Aroeira-salso & $\mathrm{e}$ & 45 & 0,62 \\
\hline Schinus terebinthifolius Raddi & Aroeira-pimenteira & $\mathrm{n}$ & 17 & 0,24 \\
\hline Annonaceae & & & & \\
\hline $\begin{array}{l}\text { Annona squamosa L. } \\
\text { Apocynaceae }\end{array}$ & Fruta-do-conde & e & 4 & 0,06 \\
\hline Allamanda cathartica $L$. & Alamanda & e & 1 & 0,01 \\
\hline Nerium oleander $\mathrm{L}$. & Loandro & e & 41 & 0,57 \\
\hline $\begin{array}{l}\text { Plumeria rubra L. } \\
\text { Thevetia thevetioides (Kunth) K. Schum }\end{array}$ & Jasmim-manga & e & 17 & 0,24 \\
\hline Adans & Chapéu-de-Napoleão & e & 4 & 0,06 \\
\hline Araliaceae & & & & \\
\hline Dizygotheca elegantissima ( Veitcyh. Ex & & & & \\
\hline Mast.) R. Vig \& Guill. & Falsa-aralia & e & 1 & 0,01 \\
\hline Schefflera actinophylla (Endl.) Harms & Chifre-de-veado & e & 17 & 0,24 \\
\hline Schefflera arboricola Hayata & Cheflera & $\mathrm{e}$ & 6 & 0,08 \\
\hline Araucariaceae & & & & \\
\hline Araucaria angustifolia (Bertol.) Kuntze & Pinheiro-do-paraná & $\mathrm{n}$ & 4 & 0,06 \\
\hline $\begin{array}{l}\text { Araucaria columnaris Hook. } \\
\text { Arecaceae }\end{array}$ & Araucária-excelsa & e & 3 & 0,04 \\
\hline $\begin{array}{l}\text { Acrocomia aculeata (Jacq.) Lodd. ex Mart. } \\
\text { Archontophoenix cunninghamiana H. }\end{array}$ & Macaúba & e & 8 & 0,11 \\
\hline Wendl. \& Drude & Seafórtia & $\mathrm{e}$ & 34 & 0,47 \\
\hline Cayiota urens L. & Cariota & $\mathrm{e}$ & 8 & 0,11 \\
\hline $\begin{array}{l}\text { Copernicia prunifera (Mill.) H.E.Moore } \\
\text { Dypsis lutescens }\end{array}$ & Carnaúba & e & 8 & 0,11 \\
\hline (H. Wendl.) Beentje \& J. Dransf & Areca-bambu & e & 28 & 0,39 \\
\hline Euterpe edulis Mart. & Palmito-Jussara & $\mathrm{n}$ & 14 & 0,19 \\
\hline Lytocaryum weddellianum (H.Wendl.) Tol. & Coco-vedeliano & e & 14 & 0,19 \\
\hline Phoenix roebelenii O'Brien & Fênix & $\mathrm{e}$ & 1 & 0,01 \\
\hline Roystonea oleracea (NJ Jacquin) OF Cook & Palmeira-imperial & e & 74 & 1,02 \\
\hline Syagrus romanzoffiana (Cham.) Glassman & Jerivá & $\mathrm{n}$ & 36 & 0,50 \\
\hline
\end{tabular}

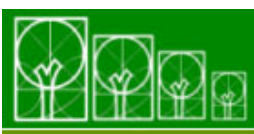




\section{Bignoniaceae}

Handroanthus chrysotrichus (Mart. ex DC.)

Mattos

Handroanthus heptaphyllus (Vell.) Mattos

Handroanthus impetiginosus (Mart. ex DC)

Mattos

Handroanthus serratifolius (Vahl)

S.O.Grose

Jacaranda mimosifolia D.Don.

Spathodea nilotica Seem

Tabebuia roseoalba (Ridl.) Sandwith

Tecoma stans (L.) Juss. ex Kunth

\section{Bixaceae}

Bixa orellana L.

\section{Cannabaceae}

Trema micrantha (L.) Blume

\section{Casuarinaceae}

Casuarina equisetifolia Forst. \& Forst.

\section{Combretaceae}

Terminalia catappa L.

Crhysobalonaceae

Licania tomentosa (Benth.) Fritsch

Cupressaceae

Cupressus lusitanica Mill.

Cupressus sempervirens L.

Juniperus chinensis L.

Thuja occidentalis L.

\section{Cycadaceae}

Cycas revoluta Thumb.

\section{Dilleneaceae}

Dillenia indica Linn.

\section{Ebenaceae}

Diospyros kaki L.F.

\section{Ericaceae}

Rhododendron simsii Planch.

\section{Euphorbiaceae}

Euphorbia cotinifolia L.

Euphorbia leucocephala Lotsy

Euphorbia pulcherrima willd ex Klotzsch

Joannesia princeps Vell.

Sapium glandulosum (L.) Morong

\section{Fabacee}

\begin{tabular}{|c|c|c|c|}
\hline Ipê-amarelo & $\mathrm{n}$ & 308 & 4,2 \\
\hline Ipê-de-folhas-pequenas & e & 5 & 0,0 \\
\hline Ipê-roxo & $\mathrm{n}$ & 102 & 1,4 \\
\hline Ipê-amarelo & $\mathrm{n}$ & 8 & 0,1 \\
\hline Jacarandá-mimoso & e & 24 & 0,3 \\
\hline Espatódea & e & 22 & 0,3 \\
\hline Ipê-branco & $\mathrm{n}$ & 30 & 0,4 \\
\hline Falso-ipê & e & 4 & 0,0 \\
\hline Urucum & e & 3 & 0,0 \\
\hline Trema & $\mathrm{n}$ & 2 & 0,0 \\
\hline Casuarina & e & 6 & 0,0 \\
\hline Chapéu-de-sol & e & 47 & 0,6 \\
\hline Oiti & e & 11 & 0 \\
\hline Cedro & e & 18 & 0,2 \\
\hline Cipreste-italiano & e & 6 & 0,0 \\
\hline Zimbro & e & 8 & 0 \\
\hline Tuia & e & 10 & \\
\hline Cica & e & 1 & 0, \\
\hline Árvore-do-dinheiro & e & 1 & 0.0 \\
\hline Caqui & e & 5 & 0,0 \\
\hline Azaléia & e & 77 & 1 \\
\hline Leiteiro-vermelho & e & 6 & \\
\hline Neve-da-montanha & e & 2 & \\
\hline Poinséttia & e & 14 & 0 , \\
\hline Boleira & e & 6 & \\
\hline Leiteiro & $\mathrm{n}$ & 3 & \\
\hline
\end{tabular}

Acacia podalyriifolia A. Cunningham ex G.

Don.

Anadenanthera colubrina (Vell.) Brenan

Anadenanthera peregrina (L.) Speg.

Andira anthelmia (Vell.) F.F.Macbr

Bauhinia divaricata Lam.

Bauhinia forficata Link

Caesalpinia echinata Lam.

Caesalpinia pulcherrima (L.) Sw.

$\begin{array}{cccc}\text { Acácia } & \mathrm{e} & 2 & 0,03 \\ \text { Angico-branco } & \mathrm{n} & 8 & 0,11 \\ \text { Angico-vermelho } & \mathrm{n} & 1 & 0,01 \\ \text { Angelim-amargoso } & \mathrm{n} & 1 & 0,01 \\ \text { Pata-de-vaca } & \mathrm{e} & 113 & 1,56 \\ \text { Pata-de-vaca } & \mathrm{n} & 2 & 0,03 \\ \text { Pau-Brasil } & \mathrm{e} & 7 & 0,10 \\ \text { Flamboianzinho } & \mathrm{e} & 78 & 1,08\end{array}$


Calliandra houstoniana (Mill.) Standl. Cassia fistula L.

Cassia javanica L.

Cassia leptophylla Vogel

Delonix regia (Bojer ex Hook.) Raf.

Erythrina crista-galli L.

Gleditsia triacanthos L.

Hymenaea courbaril L.

Inga marginata Willd.

Inga vera Willd.

Leucaena leucocephala (Lam.) de Wit

Leucochloron incuriale (Vell.) Barneby \& J.W.Grimes

Libidibia ferrea (Mart. ex Tul.) L.P.Queiroz

Machaerium hirtum (Vell.) Stellfeld

Machaerium villosum Vogel

Peltophorum dubium (Spreng.) Taub.

Pithecellobium dulce (Roxb.) Benth.

Platycyamus regnellii Benth.

Poincianella pluviosa (DC.) L.P.Queiroz

Pterocarpus violaceus Vogel

Samanea tubulosa (Benth.) Barneby \&

J.W.Grimes

Schizolobium parahyba (Vell.) S. F.Blake

Senna didymobotrya (Fresen.) HS Irwin \&

Barneby

Tipuana tipu (Benth.) Kuntze

\section{Griseliniaceae}

Pittosporum tobira (Thunb.) W. T. Aiton

\section{Lauraceae}

Ocotea pulchella (Nees \& Mart.) Mez

Persea americana Mill.

\section{Lecythidaceae}

Cariniana estrellensis (Raddi) Kuntze

Cariniana legalis (Mart.) Kuntze

\section{Lytrhaceae}

Lafoensia pacari A.St.-Hil.

Lagerstroemia indica L.

Lagerstroemia speciosa (L.) Pers.

Punica granatum L.

\section{Magnoliaceae}

Magnolia champaca (L.) Baill. ex Pierre.

Magnolia grandiflora $\mathrm{L}$.

Malpighiacee

Byrsonima sp.

Malpighia emarginata DC.

\section{Malvaceae}

Ceiba erianthos (Cav.) K.Schum.

Hibiscus rosa-sinensis $\mathrm{L}$.

Luehea grandiflora Mart.

Pachira aquatica Aubl.

\begin{tabular}{|c|c|c|c|}
\hline Caliandra & e & 18 & 0,25 \\
\hline Cássia-chuva-de-ouro & e & 17 & 0,24 \\
\hline Cassia-rosea & e & 45 & 0,62 \\
\hline Falso-Barbatimão & e & 57 & 0,79 \\
\hline Flamboyant & e & 39 & 0,54 \\
\hline Murungu & $\mathrm{n}$ & 6 & 0,08 \\
\hline Espinheiro-da-virginia & e & 2 & 0,03 \\
\hline Jatobá & $\mathrm{n}$ & 1 & 0,01 \\
\hline Ingá & $\mathrm{n}$ & 3 & 0,04 \\
\hline Ingá-do-brejo & $\mathrm{n}$ & 4 & 0,06 \\
\hline Leocena & e & 3 & 0,04 \\
\hline Chico-Pires & $\mathrm{n}$ & 1 & 0,01 \\
\hline Pau-ferro & $\mathrm{n}$ & 4 & 0,06 \\
\hline Jacarandá & $\mathrm{n}$ & 1 & 0,01 \\
\hline Jacarandá-paulista & $\mathrm{n}$ & 23 & 0,32 \\
\hline Canafistula & $\mathrm{n}$ & 8 & 0,11 \\
\hline Grumachil & e & 1 & 0,01 \\
\hline Pau-pereira & $\mathrm{n}$ & 8 & 0,11 \\
\hline Sibipiruna & e & 239 & 3,31 \\
\hline Aldrago & e & 2 & 0,03 \\
\hline Alforobo & e & 3 & 0,04 \\
\hline Guapuruvú & $\mathrm{n}$ & 1 & 0,01 \\
\hline Cássia-africana & e & 1 & 0,01 \\
\hline Tipuana & e & 11 & 0,15 \\
\hline Pitosporo & e & 5 & 0,07 \\
\hline Canela-preta & $\mathrm{n}$ & 2 & 0,03 \\
\hline Abacate & e & 16 & 0,22 \\
\hline Jequitibá-rosa & $\mathrm{n}$ & 2 & 0,03 \\
\hline Jequitibá & $\mathrm{n}$ & 4 & 0,06 \\
\hline Dedaleiro & $\mathrm{n}$ & 4 & 0,06 \\
\hline Resedá & e & 1708 & 23,64 \\
\hline Resedá-gigante & e & 231 & 3,20 \\
\hline Romã & e & 40 & 0,55 \\
\hline Magnolia-amarela & e & 89 & 1,23 \\
\hline Magnóia & e & 7 & 0,10 \\
\hline Murici & e & 2 & 0,03 \\
\hline Acerola & e & 32 & 0,44 \\
\hline Paineira & $\mathrm{n}$ & 9 & 0,12 \\
\hline Hibisco & e & 179 & 2,48 \\
\hline Açoita-cavalo & $\mathrm{n}$ & 1 & 0,01 \\
\hline Castanha-do-Maranhão & e & 3 & 0,04 \\
\hline
\end{tabular}




\section{Melatomataceae}

Brunfelsia uniflora (Pohl) D. Don

Tibouchina candolleana Cogn.

Tibouchina granulosa (Desr.) Cogn.

\section{Meliaceae}

Cedrela fissilis Vell.

\section{Moraceae}

Ficus benjamina $\mathrm{L}$.

Ficus insipida Willd.

Ficus microcarpa L. F.

Maclura tinctoria (L.) D.Don ex Steud.

Morus nigra L.

Muntingiaceae

Muntingia calabura L.

Myrsinaceae

Myrsine guianensis (Aubl.) Kuntze

Myrtaceae

Callistemon imperiallis (Sol. Ex Gaertn.) G.

Don ex Loud.

Eucalyptus sp.

Eugenia uniflora L.

Eugenia uvalha Cambess.

Myrcia sp.

Myrcia splendens (Sw.) DC.

Myrciaria cauliflora (Mart.) O. Berg.

Psidium cattleianum Sabine

Psidium guajava $\mathrm{L}$.

Psidium sp.

Syzygium cumini (L.) Skeels

Syzygium jambos (L.) Alston

Nyctaginaceae

Bougainvillea glabra Choisy

Oleraceae

Jasminum primulinum Hemsl.

Ligustrum lucidum W.T.Aiton

\section{Oxalidaceae}

Averrhoa carambola L.

\section{Pinaceae}

Pinus elliottii L.

\section{Podocarpaceae}

Podocarpus macrophyllus (Thumb.) D. Don

\section{Polygonaceae}

Triplaris americana L.

Proteaceae

Grevillea robusta A. Cunn. Ex. R. Br.

Rhamnaceae

Hovenia dulcis Thunb.

\section{Rosaceae}

Eriobotrya japonica (Thunb.) Lindl.

Prunus persica ( L.) Batsch

Prunus serrulata Lindl.

\begin{tabular}{|c|c|c|c|}
\hline Manacá-de-jardim & $\mathrm{e}$ & 28 & 0,39 \\
\hline Manacá-da-serra & $\mathrm{n}$ & 28 & 0,39 \\
\hline Quaresmeira & $\mathrm{n}$ & 168 & 2,32 \\
\hline Cedro-rosa & $\mathrm{n}$ & 2 & 0,03 \\
\hline Ficus-benjamina & $\mathrm{e}$ & 92 & 1,27 \\
\hline Figueira-do brejo & $\mathrm{n}$ & 1 & 0,01 \\
\hline Fícus & e & 1 & 0,01 \\
\hline Maclura & $\mathrm{n}$ & 1 & 0,01 \\
\hline Amora & e & 94 & 1,30 \\
\hline Calabura & $\mathrm{n}$ & 1 & 0,01 \\
\hline Capororoca & $\mathrm{n}$ & 5 & 0,07 \\
\hline Escova-de-garrafa & e & 169 & 2,34 \\
\hline Eucalipto & e & 1 & 0,01 \\
\hline Pitanga & $\mathrm{n}$ & 155 & 2,15 \\
\hline Uvaia & $\mathrm{n}$ & 12 & 0,17 \\
\hline Guamixama & e & 4 & 0,06 \\
\hline Guamirim & $\mathrm{n}$ & 4 & 0,06 \\
\hline Jaboticaba & $\mathrm{n}$ & 4 & 0,06 \\
\hline Araçá & $\mathrm{n}$ & 13 & 0,18 \\
\hline Goiaba & $\mathrm{n}$ & 51 & 0,71 \\
\hline Guamixama & e & 1 & 0,01 \\
\hline Jambolão & e & 4 & 0,06 \\
\hline Jambo & e & 2 & 0,03 \\
\hline Primavera & $\mathrm{n}$ & 20 & 0,28 \\
\hline Jasmim-amarelo & e & 1 & 0,01 \\
\hline Alfineiro & e & 113 & 1,56 \\
\hline Carambola & e & 8 & 0,11 \\
\hline Pinus & e & 6 & 0,08 \\
\hline Podocarpus & e & 8 & 0,11 \\
\hline Pau-de-formiga & e & 10 & 0,14 \\
\hline Grevilha & e & 9 & 0,12 \\
\hline Uva-japonesa & e & 7 & 0,10 \\
\hline Nêspera & e & 27 & 0,37 \\
\hline Pêssego & e & 2 & 0,03 \\
\hline Cerejeira-do-Japão & e & 27 & 0,37 \\
\hline
\end{tabular}




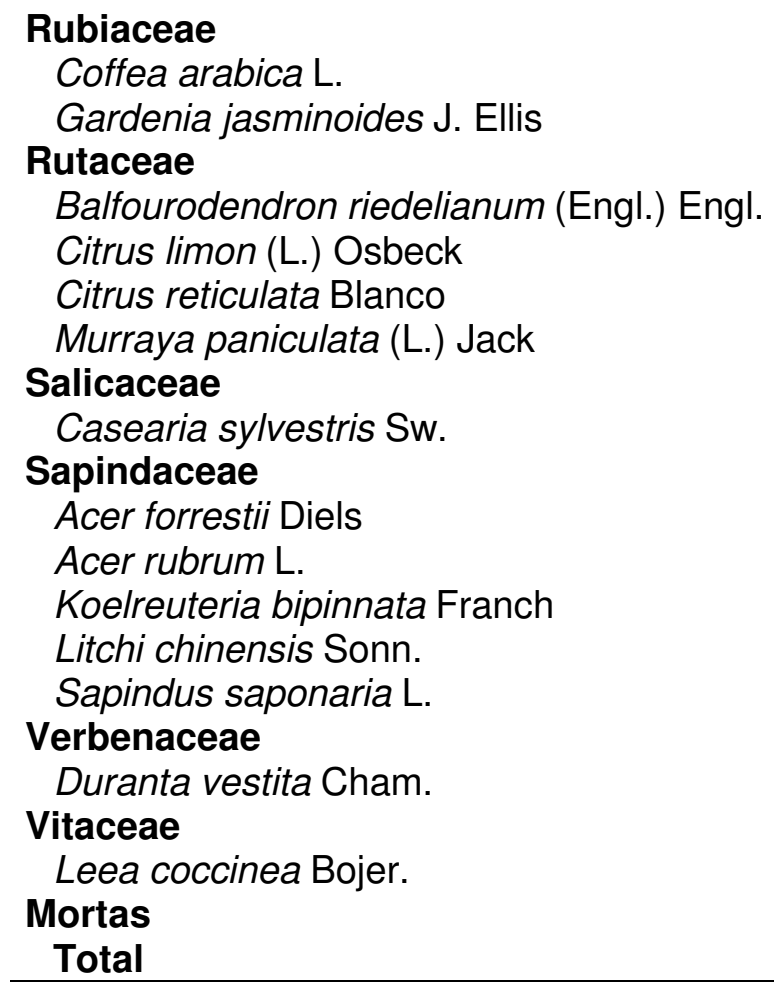

\begin{tabular}{cccc} 
Café & e & 8 & 0,11 \\
Gardenia & e & 3 & 0,04 \\
Pau-marfim & & & \\
Limão & $\mathrm{n}$ & 3 & 0,04 \\
Mexerica & $\mathrm{e}$ & 22 & 0,30 \\
Murta & $\mathrm{e}$ & 11 & 0,15 \\
Erva-de-lagarto & $\mathrm{e}$ & 1362 & 18,85 \\
& $\mathrm{n}$ & 3 & 0,04 \\
Ácer-negundo & $\mathrm{e}$ & 2 & 0,03 \\
Acer-vermelho & $\mathrm{e}$ & 1 & 0,01 \\
Árvore-da-China & $\mathrm{e}$ & 28 & 0,39 \\
Lichia & $\mathrm{e}$ & 3 & 0,04 \\
Saboeiro & $\mathrm{e}$ & 5 & 0,07 \\
& & & \\
Pingo-de-ouro & $\mathrm{e}$ & 56 & 0,77 \\
& & & \\
Léia & $\mathrm{e}$ & 1 & 0,01 \\
& & 36 & 0,50 \\
& & 6829 & 100 \\
\hline
\end{tabular}

Algumas das espécies exóticas encontradas podem causar prejuízos ecológicos e econômicos incalculáveis, como é o caso da murta (Murraya paniculata) que é um vetor para agentes que prejudicam espécies de citrus como a laranja, limão e mexerica, muitas vezes provocando a morte destas espécies que possuem alto valor agropecuário no município (ZHOU; GABRIEL, 2007). Ainda, algumas caracterizam como invasoras, como o falso-ipê (Tecoma stans) e a uva-japonesa (Holvenia dulcis), que invadem áreas e competem com espécies nativas.

Muitas espécies nativas poderiam ser utilizadas para a arborização, fornecendo alimento para a fauna, aumentando a possibilidade de trocas genéticas entre fragmentos próximos, também evitaria que plantas invasoras e competidoras causassem prejuízos para o ambiente local e evitaria a extinção de espécies nativas. Algumas espécies nativas e frutíferas se adaptam muito bem à arborização urbana, como exemplo, acerola (Malpighia emarginata), Araçá (Psidium cattleyanum) aroeira, (Schinus terebinthifolius), pitangueira (Eugenia uniflora) e ingazeiro (Inga edulis), indicados para a arborização urbana por Valaski et al. (2008) em Curitiba.

Muitos trabalhos por toda a parte do mundo defendem o uso da arborização urbana com espécies nativas e frutíferas para o alimento não somente da fauna, mas também para a alimentação humana. Carvalho et al. (2010) encontraram em alguns bairros de Curitiba cerca de $57 \%$ do total de árvores como sendo úteis para este fim, sendo principalmente, (4) 
jerivá (Syagrus romanzoffiana), o pinheiro (Araucaria angustifolia) e a pitangueira (Eugenia uniflora). Manica (1997) cita Porto Alegre; Mascaró e Mascaró (2003) acrescentam o exemplo de Sevilha e Córdoba no uso de espécies nativas e frutíferas. Mello Filho (1985) apresentou exemplos bem sucedidos de arborização com macieiras e pereiras na Suíça e laranjeiras em Assunção (Paraguai) e relaciona o sucesso do empreendimento ao grau de cultura da população. Ferreira (1985) indica algumas espécies que podem ser aproveitadas na arborização das ruas como a romãzeira anã, goiabeira e nespereira. Penteado (1968) mensiona a cidade de Belém, que é chamada de cidade verde ou das mangueiras, graças ao projeto de arborização posto em prática a partir do início do século passado.

Há uma grande concentração de indivíduos de algumas espécies assim como Largestroemia indica com $25 \%$ dos indivíduos e Murraya paniculata (20\%). Tal observação demonstra a falta de planejamento no viveiro de mudas do horto municipal. De acordo com as recomendações de Grey e Deneke (1978), apud Milano e Dalcin (2000), cada espécie não deve ultrapassar $10-15 \%$ do total de indivíduos da população arbórea, para um bom planejamento da arborização urbana. Segundo Santamour-Júnior (2002) grande diversidade de espécies de árvores na paisagem urbana é necessária justamente para garantir o máximo de proteção contra pragas e doenças, evitando assim, o aniquilamento de espécies, onde a arborização é muito homogênea.

Dessa forma, segundo o mesmo autor, recomenda-se não exceder mais que $10 \%$ da mesma espécie, $20 \%$ de um mesmo gênero e $30 \%$ de uma família botânica. Muitos indivíduos estão fora deste padrão no município de Socorro, onde 18 espécies possuem mais de $10 \%$ do número de indivíduos. A espécie Murraya paniculata, além de ser a segunda mais freqüente, apresenta ainda um adicional na problemática, foi proibido por lei municipal e estadual o transporte, produção, plantio e comercialização e terá que ser substituída, por razão de ser danosa aos cítrus como citado acima. Os resedás, espécie mais freqüente no município, se mostraram muito aptas a serem plantadas sob fiação e em calçamento, pois é de tamanho médio, não atrapalham a fiação e com raízes geralmente subterrâneas.

Danos - foram registrados 36 espécies, ou seja, 24,3\% do total, que estavam causando algum tipo de dano à infraestrutura urbana (Tabela 3). Foram registradas 1151 agressões, ou seja, 8,43\% do total das agressões possíveis, isso porque dos 6829 indivíduos encontrados cada um poderia causar dois tipos de agressão, fiação e calçamento, sendo no total, 13658 agressões possíveis. Ao calçamento foram registradas

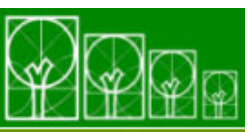

$\mathbf{S} \cdot \mathbf{B} \cdot \mathbf{A} \cdot \mathbf{U}$ Soc. Bras. de Arborização Urbana 
351 agressões de nível dois, 91 de nível três, quatro de nível quatro e nenhuma de nível cinco, somando um total de 446 agressões (Tabela 4).

Tabela 3. Espécies registradas em levantamento na arborização urbana do município de Socorro - SP, como causadoras de danos, ordenadas de forma decrescente por ID, que é índice de danos. $\mathrm{Cr}$ - Dano relativo ao calçamento e $\mathrm{Fr}$ - Dano relativo à fiação

Table 3. Survey of species used in the urban forest of Socorro, SP, sorted by decreasing order of ID, which is an index of damage. $\mathrm{Cr}$ - Damage on the pavement and $\mathrm{Fr}$ Damage on the wiring

\begin{tabular}{lrrr}
\hline \multicolumn{1}{c}{ Nome científico } & Cr & Fr & ID \\
& & & \\
\hline Hovenia dulcis & 21,44 & 7,55 & 14,49 \\
Jacaranda mimosifolia & 5,00 & 12,26 & 8,63 \\
Cassia leptophylla & 9,22 & 7,15 & 8,18 \\
Poincianella pluviosa & 7,91 & 6,25 & 7,08 \\
Handroanthus heptaphyllus & 9,00 & 4,53 & 6,77 \\
Ligustrum lucidum & 6,51 & 5,68 & 6,09 \\
Terminalia catappa & 5,43 & 6,10 & 5,76 \\
Ocotea pulchella* & 0,00 & 11,32 & 5,66 \\
Bauhinia divaricata & 7,64 & 3,21 & 5,42 \\
Lagerstroemia speciosa & 4,16 & 4,61 & 4,38 \\
Magnolia champaca & 4,30 & 3,73 & 4,02 \\
Tipuana tipu & 6,14 & 0,00 & 3,07 \\
Leucaena leucocephala* & 0,00 & 5,03 & 2,52 \\
Koelreuteria bipinnata & 3,22 & 1,08 & 2,15 \\
Handroanthus impetiginosus & 4,05 & 0,22 & 2,13 \\
Lagerstroemia indica & 0,24 & 3,53 & 1,89 \\
Spathodea nilotica & 0,00 & 2,74 & 1,37 \\
Schefflera arboricola & 0,00 & 2,52 & 1,26 \\
Cassia javanica & 2,50 & 0,00 & 1,25 \\
Schinus mole & 0,67 & 1,17 & 0,92 \\
Ficus benjamina & 0,90 & 0,90 & 0,90 \\
Callistemon imperiallis & 0,71 & 1,03 & 0,87 \\
Tibouchina granulosa & 0,71 & 0,94 & 0,83 \\
Citrus reticulata & 0,00 & 1,37 & 0,69 \\
Licania tomentosa & 0,00 & 1,37 & 0,69 \\
Persea americana & 0,00 & 0,94 & 0,47 \\
Schinus terebinthifolius & 0,00 & 0,89 & 0,44 \\
Cupressus lusitanica & 0,00 & 0,84 & 0,42 \\
Roystonea oleracea & 0,00 & 0,61 & 0,31
\end{tabular}


Eriobotrya japonica

\begin{tabular}{lll}
0,00 & 0,56 & 0,28 \\
0,17 & 0,37 & 0,27 \\
0,00 & 0,48 & 0,24 \\
0,00 & 0,39 & 0,19 \\
0,10 & 0,24 & 0,17 \\
0,00 & 0,34 & 0,17 \\
0,00 & 0,04 & 0,02 \\
\hline
\end{tabular}

Handroanthus chrysotrichus

Morus nigra

Delonix regia

Eugenia uniflora

Hibisco rosa-sinensis

0,00

* Indivíduos com menos de 5 indivíduos

Tabela 4. Indivíduos registrados em levantamento de espécies utilizadas na arborização urbana do município de Socorro - SP, como causadores de dano à estrutura, sendo estes calçamento e a fiação, seguidos de seus devidos níveis, variando de

1 à 5 , sendo 1 ausência de danos e 5 danos máximos

Table 4. Individuals registered in the list of species used in urban greening of Socorro - SP, causing damage to structure, which are pavement and wiring, followed by their proper levels, ranging from 1 to 5 , with 1 being no damage and 5 maximum damage

\begin{tabular}{lccccc|ccccc}
\hline Estrutura & \multicolumn{5}{c|}{ Calçamento } & \multicolumn{5}{c}{ Fiação } \\
Nível & $\mathbf{1}$ & $\mathbf{2}$ & $\mathbf{3}$ & $\mathbf{4}$ & $\mathbf{5}$ & $\mathbf{1}$ & $\mathbf{2}$ & $\mathbf{3}$ & $\mathbf{4}$ & $\mathbf{5}$ \\
Total & 6347 & 351 & 91 & 4 & 0 & 6088 & 514 & 189 & 2 & 0 \\
\hline
\end{tabular}

As dez espécies que causaram danos ao calçamento somaram $82,58 \%$ do total de danos a esta estrutura, sendo estas: Hovenia dulcis (21,44\%), Cassia leptophylla (9,22\%), Handroanthus heptaphyllus $(9,00 \%)$, Poincianella pluviosa $(7,91 \%)$, Bauhinia divaricata (7,64\%), Ligustrum lucidum (6,51\%), Tipuana tipu (6,14\%), Terminalia catappa (5,43\%), Jacaranda mimosifolia (5,00\%) e Magnolia champaca (4,30\%). À fiação foram registradas 514 agressões de nível dois, 189 de nível três, dois de nível quatro e nenhuma de nível cinco, totalizando 705 agressões (Tabela. 4). As dez espécies que causaram algum dano à fiação somaram $70,48 \%$ do total e foram: Jacaranda mimosifolia (12,26\%), Ocotea pulchella (11,32\%), Hovenia dulcis (7,55\%), Cassia leptophylla (7,15\%), Poincianella pluviosa (6,25\%), Terminalia catappa (6,10\%), Ligustrum lucidum (5,68\%), Leucaena leucocephala (5,03\%), Lagerstroemia speciosa (4,61\%) e Handroanthus heptaphyllus (4,53\%). As dez espécies que representaram maior valor de danos somaram $72,5 \%$ do total e as espécies foram: Hovenia dulcis (14,49), seguida de Jacaranda mimosifolia (8,63\%), Cassia leptophylla (8,18\%), Poincianella pluviosa (7,08\%), Handroanthus heptaphyllus (6,77\%), Ligustrum Iucidum (6,09\%), Terminalia catappa (5,76\%) Ocotea pulchella (5,66\%), Bauhinia divaricata $(5,42 \%)$ e Lagerstroemia speciosa (4,38\%).

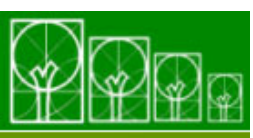

$\mathbf{S} \cdot \mathbf{B} \cdot \mathbf{A} \cdot \mathbf{U}$ Soc. Bras. de Arborização Urbana 
No município de Águas de São Pedro, SP, com 3640 km² de proporções 123 vezes menor que o município de Socorro, que possui $448.000 \mathrm{Km}^{2}$ (IBGE, 2009), em um levantamento na área urbana realizada por Bortoleto et al. (2007), semelhante a este, foram encontrados pouco menos que $50 \%$ de indivíduos e 13 espécies a mais, sendo que nenhuma possuía mais que 500 indivíduos, mostrando um planejamento muito mais adequado, com grande quantidade de indivíduos, equitabilidade e riqueza de espécies. Porém este mesmo trabalho constatou que muitas das espécies são exóticas em Águas de São Pedro, possuindo pouco menos de $40 \%$ de espécies nativas.

Das 36 espécies registradas como agressoras de alguma forma, muitas delas ocorreram por falta de planejamento para os locais onde são plantados e cuidados com poda. As podas são feitas, geralmente, no município, uma vez por ano e de forma agressiva para o indivíduo, havendo corte muito baixo, geralmente na altura das primeiras folhas, deixando apenas galhos com mais até $5 \mathrm{~cm}$ de diâmetro desfolhados. Porém este fato é comum em outros locais. Volpe-Filik et al. (2007) observaram problemas em $69 \%$ das podas efetuadas em Piracicaba, no bairro São Dimas, evidenciando um manejo inadequado por parte da Prefeitura ou pela população, embora exista uma Lei Municipal que proíbe o munícipe de efetuar as podas. Segundo os autores, um manejo inadequado, principalmente em relação as podas mal feitas, proporciona tocos e lascas no tronco, facilitando a entrada de patógenos e insetos e em galhos grossos, evidencia o mau trato em relação às árvores.

Quanto ao calçamento, em muitas situações já foi comprovado o uso de estruturas que evitam o crescimento das raízes para fora do calçamento, assim como manilhas, porém isto torna a planta mais suscetível à queda por ventos ou colisões. Porém, é muito importante observar muito bem o espaçamento antes de ser feito o plantio e utilizar covas de tamanhos corretos podendo evitar assim o uso de estruturas adjacentes. O mais adequado é evitar algumas espécies como Hovenia dulcis, Jacaranda mimosifolia, Cassia leptophylla, Poincianella pluviosa, Handroanthus heptaphyllus, Ligustrum lucidum, Terminalia catappa e Bauhinia divaricata em calçamentos estreitos, principalmente quando houver muros e casas próximas, evitando danificações. Os danos causados pelas raízes das árvores em calçadas, sarjetas e esgotos são, em média, 25\% do custo de manutenção anual com árvores urbanas e, pelas cifras que atingem, esses problemas multimilionários precisam ser mais bem estudados (MCPHERSON; PEPER, 1996). Em Piracicaba, VolpeFilik et al. (2007), registraram que 37\% dos indivíduos possuíam raízes aparentes e quase todos afetando o calçamento.

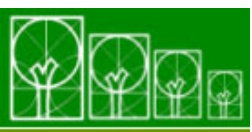

$\mathbf{S} \cdot \mathbf{B} \cdot \mathbf{A} \cdot \mathbf{U}$ Soc. Bras. de Arborização Urbana 
Quanto à fiação, muitas cidades como Curitiba-PR, Rio de Janeiro-RJ, Maringá-PR, Manaus-AM, além dos bairros de São Paulo-SP, comprovam que é possível possuir árvores de grande porte nos centros urbanos, porém há de se tomar muitas precauções durante o ano todo, fazendo cortes em "v" ou mesmo utilizando de podas técnicas, para deixar a fiação passar pelo meio das árvores e retirando galhos mais velhos para evitar quedas. Um exemplo de planejamento com espécies tidas de risco é o caso do Abricó-de-macaco (Couroupita guianensis) no município do Rio de Janeiro, onde muitas árvores com frutos grandes, com mais de dois quilogramas quando maduros e copas com mais de 8 metros de altura, porém não destroem fiação por serem tomadas precauções adequadas nas podas tipo "v" e seus frutos serem retirados ainda maduros para evitar quedas e acidentes.

Segundo Rocha et al. (2004), no município do Rio de Janeiro, as espécies chapéude-sol, cássia e flamboyant, não estão sendo indicadas para arborização de ruas. A primeira espécie possui folhas grandes, que nos meses de julho e agosto se desprendem das árvores, sujando as calçadas e ruas, além de causar entupimento da rede pluvial (bocas-delobo). Cássia é uma espécie de rápido crescimento, cuja madeira é de baixa densidade e, conseqüentemente, baixa resistência mecânica, quebrando com facilidade, o que pode causar transtornos devido à queda de galhos, esta espécie é facilmente encontrada no município de Socorro, principalmente em ruas movimentadas. Flamboyant não é indicado para arborização de ruas, por possuir raízes superficiais que podem danificar as calçadas, porém podem ser utilizadas em áreas verdes e canteiros centrais, onde há maior espaçamento. Além disso, Coutinho et al. (1998) constataram a presença significativa do Cerambicídeo Oncideres saga, conhecido como serrador, em árvores de flamboyant da arborização do Campus da UFRRJ, em Seropédica, município vizinho ao de Nova Iguaçu.

Algumas espécies, assim como Ficus benjamina, possuem uma forte tendência a problemas futuros, isso por ser uma espécie que cresce muito e possui raízes tabulares, que crescem para os lados, inclusive com exposição para fora da terra, para melhor estabilidade da planta. Rocha et al. (2004) em um trabalho realizado em Nova Iguaçu, RJ, mostraram que esta espécie é a mais abundante em alguns bairros do município e concluiu que é inadequado para a arborização de vias públicas devido à incompatibilização com estruturas urbanas, assim também o flamboyant, cássia e chapéu-de-sol, registrados no município e apresentando danos, principalmente ao calçamento. Santana e Santos (1999) comentaram que o gênero Ficus é causador de problemas na arborização do Campus da Universidade Estadual de Feira de Santana (UEFS).

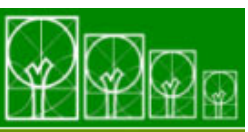

$\mathbf{S} \cdot \mathbf{B} \cdot \mathbf{A} \cdot \mathbf{U}$ Soc. Bras. de Arborização Urbana 
O índice mostra-se bem preciso quanto às espécies que mais causam danos, dando valores a elas e podendo classificadas ordenadamente, sendo mais preciso do que se apenas houvesse uma citação das espécies que causam danos, porém é aconselhável que esta classificação em níveis seja feita sempre pela mesma pessoa durante o trabalho todo, ou mesmo um grupo de pessoas, sendo sempre as mesmas durante o trabalho e nesse caso deve ser feito uma média de cada valor.

\section{CONCLUSÕES}

O município de Socorro precisa de uma grande reestruturação em sua arborização. Contando com as espécies sugeridas a serem cortadas, árvores mortas e murtas (Murraya paniculata), que terão que ser retiradas por Lei Estadual e Municipal, quase 1500 árvores precisam ser substituídas. Além do mais, muitas espécies podem ser plantadas nas ruas do município, sendo assim seria interessante fazer um grande projeto de arborização, com a participação de ONGs e de escolas. Os resedás não devem ser usados no município, pois apresentam $25 \%$ das árvores plantadas no local são desta espécie.

Os danos quanto à fiação podem ser resolvidos, em quase todos os casos, com um programa de podas adequadas, promovendo cursos para funcionários da CPFL e da Prefeitura. Quanto ao calçamento, em muitos casos, reestruturação do calçamento resolve, porém há de ser feito um planejamento com as espécies e o espaçamento para que não haja mais danos.

Menos de $50 \%$ da capacidade de suporte de vegetação vem sendo utilizada, e ainda podem ser plantados mais 7226 indivíduos, de portes variados. Tal ação usada de forma correta poderia contribuir para "grande reserva ecológica", que tornaria a vida dos seres humanos e animais possível e agradável. No município há muitas espécies exóticas e isso deveria ser revisto com a reestruturação da arborização urbana, promovendo a inclusão de espécies nativas, aumentando assim, a diversidade e a equitabilidade, ou seja, a relação de frequência entre espécies. 


\section{AGRADECIMENTOS}

Ao amigo e estudante de engenharia Marcos Magon por auxiliar com os mapas e orientações sobre bairros e ruas da cidade. Ao amigo Dervino Dermino Santin, por auxiliar na identificação de muitas espécies e Isabel Machado Guigon de Araújo pelo auxílio na revisão do trabalho.

\section{REFERÊNCIAS}

APG III. 2009. An update of the Angiosperm Phylogeny Group classification for the orders and families of flowering plants: APG III. Botanical Journal of the Linnean Society 161:105-121.

BORTOLETO, S.; SILVA FILHO, D. F.; SOUZA, V. C.; FERREIRA, A. P.; POLIZEL, J. L.; RIBEIRO, R. C. S.; Composição e distribuição da arborização viária da estância de Águas de São Pedro - SP. Revista da Sociedade Brasileira de Arborização Urbana, Piracicaba - SP, v 2, n 3, 2007.

BRUMMIT, R.K. \& POWELL, C.E. Authors of plant names. Royal Botanic Gardens, Kew. 732 p. 1992.

CARVALHO, J. A; NUCCI, J. C.; VALASKI, S.; Inventário das árvores presentes na arborização de calçadas da porção central do bairro Santa Felicidade-Curitiba/PR. Revista da Sociedade Brasileira de Arborização Urbana, Piracicaba - SP, v.5, n.1, p.126-143, 2010.

CEMIG - Companhia Energética de Minas Gerais. 2004. Manual de Arborização. Belo Horizonte - MG. 2004. 40 p.

COUTINHO, C.L; CARVAlHO, A. C.; OliVEIRA, E. S.; VEIGA, B. G. A. Oncideres saga (Dalman, 1823) (coleóptera, cerambycidae) e a arborização urbana em Seropédica, RJ. Floresta e Ambiente, v.51, p.50-54, 1998.

DOUROJEANNI, A. Dawn in the Andes, ed. ECLAC/United Nations, Santiago, Chile. 212p. 1997.

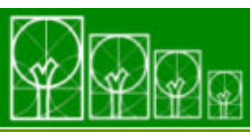

$\mathbf{S} \cdot \mathbf{B} \cdot \mathbf{A} \cdot \mathbf{U}$ Soc. Bras. de Arborização Urbana 
FERREIRA, L. A. B. Usos da vegetação In: ENCONTRO NACIONAL SOBRE ARBORIZAÇÃO URBANA, 10, 1985, Porto Alegre. Anais... Porto Alegre: Prefeitura Municipal de Porto Alegre: Secretaria Municipal do Meio Ambiente, 1985. p. 89-95.

FORZZA, R. C.; LEITMAN, P. M.; COSTA, A. F.; CARVALHO JR., A. A.; PEIXOTO, A. L.; WALTER, B. M. T.; BICUDO, C.; ZAPPI, D.; COSTA, D.P.; LLERAS, E.; MARTINELLI, G.; LIMA, H.C.; PRADO, J.; STEHMANN, J. R.; BAUMGRATZ, J. F. A.; PIRANI, J. R.; SYLVESTRE, L.; MAIA, L. C.; LOHMANN, L. G.; QUEIROZ, L. P.; SILVEIRA, M.; COELHO, M. N.; MAMEDE, M. C.; BASTOS, M. N. C.; MORIM, M. P.; BARBOSA, M. R.; MENEZES, M.; HOPKINS, M.; SECCO, R.; CAVALCANTI, T. B.; SOUZA, V. C. Introdução. in Lista de Espécies da Flora do Brasil. Jardim Botânico do Rio de Janeiro, 2010.

FOSTAD, O.; PEDERSEN, P. A. Vitality, variation, and causes of decline of trees in Oslo center (Norway). J. ARBORICULTURE 23: 155-1651997.

GOYA, C. R. Os jardins e a vegetação do espaço urbano: um patrimônio cultural. IN: II CONGRESSO BRASILEIRO DE ARBORIZAÇÃO URBANA; V ENCONTRO NACIONAL SOBRE ARBORIZAÇÃO URBANA. Anais... São Luiz: SBAU, 1994. p. 133-145.

GREY, G. W.; DENEKE, F. J. Urban forestry. New York, John Wiley, 1978, 279p.

GUZZO, P. Alterações ambientais em áreas urbanas, planejamento e legislação ambiental. In: SEMINÁRIO LATINO AMERICANO DE PLANEJAMENTO URBANO, CAMPO GRANDE/MS. Anais, 1993. p.214-222.

IBGE. 2009. Estimativas da População Brasileira. Instituto Brasileiro de Geografia e Estatística (IBGE) (14 de agosto de 2009). Disponível em <http:/www.ibge.gov.br> Página visitada em 16 de agosto de 2009.

IBGE. Censo 2010. Disponível em: <http://www.ibge.gov.br>. Acesso em: 04 out. 2011.

LIMA, A. M. L. P. Piracicaba, SP: analise da arborização viária na área central e seu entorno. Piracicaba. Tese (Doutorado) - Escola Superior de Agricultura "Luiz de Queiroz", Universidade de São Paulo, 283 p., 1993.

LORENZI, H. Árvores Brasileiras: manual de identificação e cultivo de plantas arbóreas nativas do Brasil.. $3^{\circ}$ Ed. Nova Odessa: Plantarum, 2000. 352p.

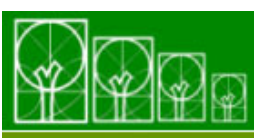

$\mathbf{S} \cdot \mathbf{B} \cdot \mathbf{A} \cdot \mathbf{U}$ Soc. Bras. de Arborização Urbana 
LORENZI, H. Árvores Brasileiras: manual de identificação e cultivo de plantas arbóreas nativas do Brasil. Vol. 2. 2ª ed. Nova Odessa: Instituto Plantarum, 384p., 2002.

MANICA, I. Fruticultura em áreas urbanas. Porto Alegre: Cinco Continentes. Editora. 154 p., 1997.

MASCARÓ, L. E. A. R. de; MASCARÓ, J. L. Vegetação urbana. Porto Alegre: L. Mascaro, 210 p., 2003.

MCPHERSON, E. G.; PEPER P. P. Costs of street tree damage to infrastructure. Arboricultural Journal, v.2O, p.143-160, 1996.

MELLO FILHO, L. E. Arborização Urbana. In: ENCONTRO NACIONAL SOBRE ARBORIZAÇÃO URBANA, 10, 1985, Porto Alegre. Anais... Porto Alegre: Prefeitura Municipal de Porto Alegre: Secretaria Municipal do Meio Ambiente, 1985. p.117-127.

MILANO, M.; DALCIN, E. Arborização de vias públicas. Rio de Janeiro: LIGHT, 226 p., 2000.

OLIVEIRA-FILHO, A. T. TreeAtlan 1.0: flora arbórea da Mata Atlântica e domínios adjacentes: um banco de dados envolvendo geografia diversidade e conservação. Available at: <http:// www.treeatlan.dcf.ufla.br/>. Access in: 10 jan. 2010.

PENTEADO, A. R. Belém - Estudo de Geografia Urbana. Tese (Livre-docência na cadeira de Geografia do Brasil) - Faculdade de Filosofia, Ciências e Letras, Universidade de São Paulo, 183f. Rio de Janeiro,1968.

SOCORRO, Prefeitura Municipal de Socorro. Disponível em: <http://www.socorro.sp.gov.br. Acesso em: 20 de agosto de 2010.

REZENDE, A. P. S. 1997. O Programa de compatibilidade da arborização urbana com redes de energia elétrica da CEMIG. In: ENCONTRO PARA CONSERVAÇÃO DA NATUREZA, 1, Viçosa: Centro Mineiro para conservação da Natureza.

ROCHA, R. T.; LELES, P. S. dos S.; OLIVEIRA-NETO, S. N. de.Arborização de vias públicas em Nova Iguaçu, RJ: o caso dos bairros Rancho Novo e Centro. Revista Árvore, Viçosa-MG, v.28, n.4, p.599-607, 2004

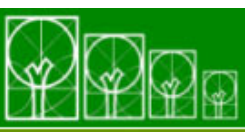

S $\cdot \mathbf{B} \cdot \mathbf{A} \cdot \mathbf{U}$ Soc. Bras. de Arborização Urbana 
SANTAMOUR JÚNIOR, F. S. Trees for urban planting: diversity unifomuty, and common sense. Washington: U.S. National Arboretum, Agriculture Research Service, 2002.

SANTANA, J. R. F.; SANTOS, G. M. M. Arborização do campus da UEFS: exemplo a ser seguido ou um grande equívoco? Sitientibus, n.20, p.103-107, 1999.

SILVA, L. M.; RODIGHIERO, D. A.; HASSE, I; CODORIN, D. A.; Arborização dos bairros Pinheiros, Brasília e Bancários em Pato Branco/PR. Scientia Agraria, Curitiba, V.9, n.3, 2008 p. $275-282$.

SMMARP. Secretaria Municipal do Meio Ambiente de Ribeirão Preto/SP. A Poda na Arborização Urbana. Apostila. 1996. 32 p.

SOS MATA ATLÂNTICA. São Paulo, 2009. Disponível em: <http://www.sosmatatlantica. org.br/>. Acesso em: 2 dez. 2009.

VALASKI, S; CARVALHO, J. A.; NUCCI, J. C. Árvore frutífera na arborização de calçadas do bairro Santa Felicidade e seus benefícios para a sociedade - Curitiba/PR Geografia. Ensino \& Pesquisa, Santa Maria: UFSM. v. 12, p. 972-985, 2008.

VELOSO, H. P.; RANGEL FILHO, A. L. \& LIMA, J. C. A. 1991. Classificação da vegetação brasileira, adaptada a um sistema universal. IBGE, Rio de Janeiro 1991.

VIDAL, M.; GONÇALVES, W. Curso de paisagismo. Viçosa, MG: UFV, 1999. 76 p.

VOLPE-FILIK , A.; SILVA, L. F., LIMA, A. M. L. P. Avaliação da arborização de ruas do bairro são Dimas na cidade de Piracicaba/sp através de parâmetros qualitativos. Revista da Sociedade Brasileira de Arborização Urbana, Piracicaba - SP. v. 2,n. 1, 2007.

ZHOU, L. J., D. W. GABRIEL, Y. P. DUAN, S. E. HALBERT, AND W. N. DIXON. 2007. First report of dodder transmission of huanglongbing from naturally infected Murraya paniculata to citrus. Notes. Plant Disease. v. 91, n. 2, 2007. 